\title{
Intergranular Oxidation Effects during Dwell-Time Fatigue of High-Strength Superalloys
}

\author{
Ulrich Krupp ${ }^{\mathrm{a}, \mathrm{c}}$, Ken Wackermann ${ }^{\mathrm{b}}$, Hans-Jürgen Christ $^{\mathrm{c}}$, \\ Magnus Hörnqvist Colliander ${ }^{\mathrm{d}}$, Krystyna Stiller ${ }^{\mathrm{d}}$ \\ ${ }^{a}$ University of Applied Sciences Osnabrück, 49076 Osnabrück, Germany \\ ${ }^{b}$ Fraunhofer Institute for Mechanics of Materials IWM, 79108 Freiburg, Germany \\ ${ }^{\circ}$ University of Siegen, 57068 Siegen, Germany \\ ${ }^{d}$ Chalmers University of Technology, 41296 Gothenburg, Sweden
}

u.krupp@hs-osnabrueck.de; ken.wackermann@iwm.fraunhofer.de; magnus.colliander@chalmers.se; hans-juergen.christ@uni-siegen.de; stiller@chalmers.se

\begin{abstract}
The present paper summarizes experimental work to identifying the mechanisms of dwell-time cracking during service operation of polycrystalline nickel-base superalloys, such as Alloy 718 and AD730. By means of crack growth monitoring during various kinds of cyclic loading in vacuum and in air using the potential drop technique, it was shown that the combination of sustained tensile stress, load reversal and oxidizing atmosphere leads to an increase in the crack-propagation rate by orders of magnitude, as compared to cyclic reference tests without dwell time and/or under vacuum conditions. By careful metallographic and theoretical analysis, the embrittling effect was attributed to stress-induced oxygen diffusion ahead of the intergranular crack tip followed by decohesion in a nanometer scale and had been termed "Dynamic Embrittlement (DE)". More recently, atom probe tomography of the near-crack tip region revealed that the damage zone consists of $\mathrm{Cr}$-rich transition oxides rather than elemental oxygen. This is in qualitative agreement with TGA measurements on alloy 718 specimens without mechanical loading, which show that crack propagation velocities of $50 \mu \mathrm{m} / \mathrm{s}$ do not allow massive $\mathrm{Cr}_{2} \mathrm{O}_{3}$ or $\mathrm{NiO}$ scale formation. By means of a quantitative analysis of the fracture surface, it became evident that grain-boundary attack depends on the grain-boundary character. This observation was supported by $4 \mathrm{pt}$ bending experiments on grain-boundary-engineered samples with a high fraction of CSL (coincident site lattice) boundaries and bicrystalline samples with well-defined grain-boundary misorientation relationships with respect to the loading axis. Taking the experimental results into account, semiquantitative modelling concepts have been developed to correlate crack propagation rates with the oxygen grainboundary diffusivity, the local microstructure and the mechanical stress states. The concepts are discussed in terms to adapt grain size and precipitate microstructure of polycrystalline superalloys.
\end{abstract}

Keywords: Grain-Boundary Diffusion, Intergranular Oxidation, Dynamic Embrittlement, Dwell-Time Fatigue

\section{INTRODUCTION}

When increasing the in-service performance of engineering metallic materials, interfaces such as grain or phase boundaries may act as the weakest links. The high energy level of their non-equilibrium state and the free volume of random high-angle grain boundaries causes grain boundaries to act as fast diffusion paths for the metallic alloying elements leading to diffusion creep (at very high temperatures) and for corrosive species. Sustained high tensile stress 
periods during cyclic operation of, e.g., aeroengines in flight operation, drives oxygen diffusion into the alloy grain boundaries. Once locally a critical oxygen or oxide concentration is exceeded, the corresponding loss in interfacial cohesion causes brittle intergranular fracture (cf. Fig. 1a). This failure mechanism, which can be considered as a hightemperature pendant to "stress corrosion cracking", has been termed "Dynamic Embrittlement (DE)" or "StressAssisted Grain Boundary Oxidation (SAGBO)", respectively (cf. [1,2]). In DE, the loss in grain boundary cohesion is due to the penetrating embrittling atoms (in a similar way than hydrogen embrittlement). It was shown to depend on the atmosphere (vacuum, air, oxygen, humidity) [3,4], the alloy grain size, the grain-boundary character and the geometrical arrangement of the grain-boundary plane with respect to the applied stress [1,5], as represented schematically in Fig. 1b. SAGBO refers rather to brittle fracture of thermally grown oxides along the grain boundaries. However, the mechanisms are still subject of controversial discussion and technical fatigue assessment relies on LCF experimental data.

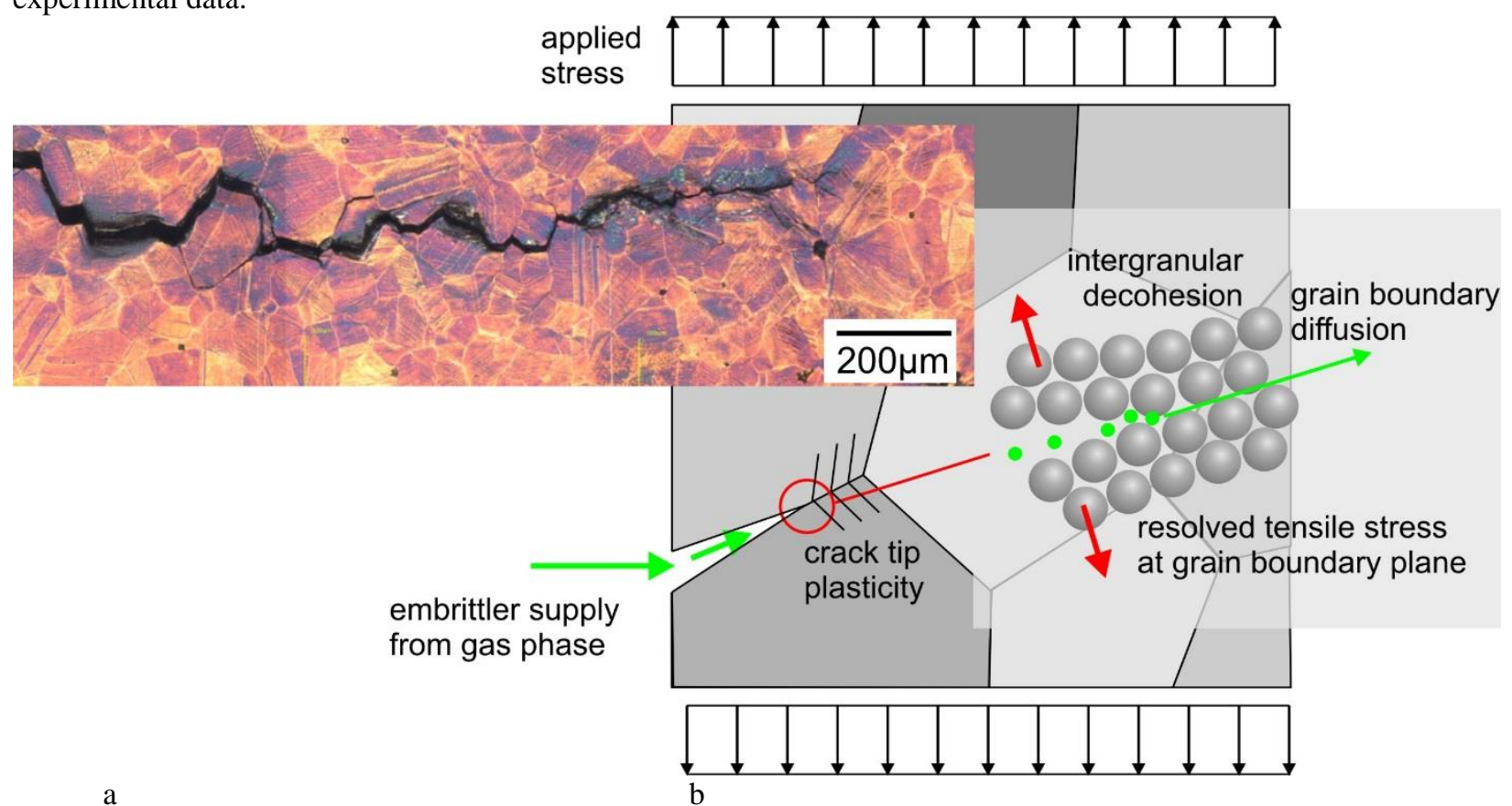

FIGURE 1. Dynamic embrittlement (DE): (a) Intergranular crack propagation in Alloy 718, and (b) schematic representation of the DE mechanism, highlighting the governing material and environment parameters.

Dwell times $\tau$ cause the fatigue crack propagation rates $d a / d N$ to increase by orders of magnitudes, leading to a transition from cycle-dependent fatigue crack propagation with the appearance of fatigue striations in the fracture surface (Fig. 2a) to time-dependent intergranular crack propagation with only very little contribution of plastic slip (Fig. 2b). In general, the cycle-dependent ( $d a / d N$ as an exponential function (Paris law) of the stress intensity range $\Delta K$, with $C$ and $m$ being material constants) and time dependent ( $d a / d t$ as a function of the stress intensity factor $K(t)$ ) contribution to fatigue crack propagation $d a / d N$ can be written as follows:

$$
\left(\frac{d a}{d N}\right)_{\text {total }}=\left(\frac{d a}{d N}\right)_{c y c l .}+\int_{0}^{\tau}\left(\frac{d a}{d t}\right) K(t) d t, \quad \text { with }\left(\frac{d a}{d N}\right)_{c y c l .}=C \Delta K^{m}
$$

Alternatively, the detrimental effect of oxidation can be accounted for by the Manson/Coffin exponential relationship between the number of cycles to fracture $N_{\mathrm{f}}$ and the applied plastic strain amplitude $\Delta \varepsilon_{\mathrm{p}} / 2$. This relationship is extended by the frequency $v$ with the exponent $k$ being a measure for the time dependence $(k=0$ : $\max$. time-dependent damage behavior; $k=1$ no time dependence) (cf. [6]):

$$
\frac{\Delta \varepsilon_{p l}}{2}=\text { const. }\left(N_{f} v^{k-1}\right)^{c} \text {. }
$$

However, time-dependent and cycle-dependent damage cannot be treated separately. It has been shown previously that dwell times cause the formation of an oxygen/oxide enriched damage zone, which is broken apart by the 
subsequent load reversal (cf. [7,8]). Furthermore, it is obvious that intergranular separation depends not only on the maximum normal stress acting on the respective grain boundary plane, but also on the grain boundary structure. Interfacial diffusivity is particular slow (i) for low-energy CSL grain boundaries with a high fraction of coincident lattices sites, and (ii) perpendicular to the tilt direction in the case of symmetrical pure tilt boundaries $[9,10]$. Therefore, the resistance to DE can be increased by increasing the fraction of CSL boundaries (including twin boundaries, cf. [9]. It is also known that the resistance can be increased by decreasing the alloy grain size. This can be attributed to the lower resolved tensile tractions acting on the grain boundaries. Since in the case of superalloys the grain size is controlled by undissolved precipitates pinning the grain boundaries during solution heat treatment, the beneficial effect of small grains during dwell-time fatigue counteracts an increase in creep strength.

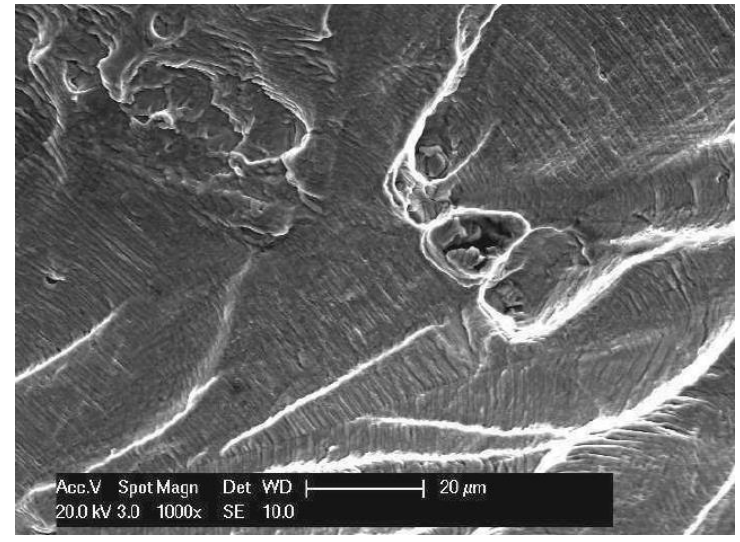

a

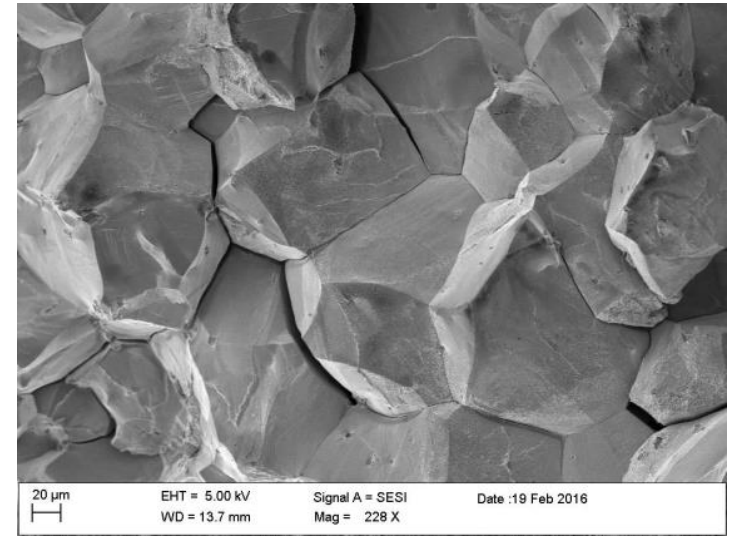

$\mathrm{b}$

FIGURE 2. Crack propagation in Alloy 718: (a) cycle dependent transgranular crack propagation manifesting itself by fatigue striations and (b) time-dependent intergranular crack propagation manifesting itself by deformation-less grain boundary facets in the fracture surface.

It is the aim of the present paper to identify the parameters governing DE and to discuss a scope for numerical modeling based on grain boundary diffusion of oxygen followed by intergranular decohesion.

\section{MATERIALS AND EXPERIMENTAL}

The study has been carried out using the two polycrystalline Ni-based superalloys Alloy 718 and AD730, the latter one being new developed by Aubert Duval for the operation at high mechanical loads at temperatures up to $700^{\circ} \mathrm{C}$. The nominal chemical composition of the materials is given in Table 1.

TABLE 1. Nominal chemical composition of the superalloys used in this study (wt.\%)

\begin{tabular}{ccccccccccccc}
\hline & Ni & Fe & Cr & Nb & Mo & Ti & Al & Co & Si & W & C & B \\
\hline Alloy 718 & bal. & 18.7 & 18.2 & 5.2 & 3.0 & 1.0 & 0.5 & 0.1 & 0.4 & -- & 0.04 & 0.004 \\
AD730 & bal. & 4 & 15.7 & 1.1 & 3.1 & 3.4 & 2.3 & 8.5 & -- & 2.7 & 0.015 & 0.01 \\
\hline
\end{tabular}

Excellent high temperature strength is obtained by heat treatment and ageing: Alloy 718 specimens were solution heat treated for $4 \mathrm{~h}$ at $1050^{\circ} \mathrm{C}$ (super-solvus) or $1000 \mathrm{C}$ (sub-solvus), respectively, followed by water quenching and ageing for $16 \mathrm{~h}\left(8 \mathrm{~h}\right.$ at $720^{\circ} \mathrm{C}$ and $8 \mathrm{~h}$ at $\left.620^{\circ} \mathrm{C}\right)$. The resulting microstructure consists of small $\gamma^{\prime \prime} \mathrm{Ni}_{3} \mathrm{Nb}$ platelets and, in the case of the sub-solvus-solutionized samples, of $\delta \mathrm{Ni}_{3} \mathrm{Nb}$ precipitates at the alloy grain boundaries (see Fig. 3a). While the $\delta$ precipitates keep the grain size small (approx. $8 \mu \mathrm{m}$ ), the super-solvus-solutionized samples became coarse grained (approx. $70 \mu \mathrm{m}-100 \mu \mathrm{m}$ ). A similar heat treatment concept was applied to AD730 specimens: super-solvus $\left(1150^{\circ} \mathrm{C}\right)$ or sub-solvus $\left(1080^{\circ} \mathrm{C}\right)$ solutionizing for $4 \mathrm{~h}$ followed by water quenching and $16 \mathrm{~h}$ ageing at $730^{\circ} \mathrm{C}$. A small alloy grain size was maintained by primary $\gamma^{\prime} \mathrm{Ni}_{3}(\mathrm{Al}, \mathrm{Ti})$ precipitates, while ageing resulted in small globular-shaped $\gamma^{\prime}$ precipitates (see Fig. 3b). 
A variety of mechanical tests were applied to identify the DE behavior under sustained load and superimposed load reversals, using SENB specimens for 4pt bending load relaxation tests and corner notch $(\mathrm{CN})$ specimens for dwelltime fatigue experiments. The specimens were heat treated and produced by turning, milling and mechanical polishing $(1 \mu \mathrm{m}$ finish). Pre-cracks were grown from a $0.25 \mathrm{~mm}$ eroded starter notch by cyclic loading at room temperature (alternatively by a thin Mo wire drawn back and forth through the notch using $6 \mu \mathrm{m}$ diamond suspension, for details see $[1,8])$ to a length of about $1.6 \mathrm{~mm}$. The subsequent crack propagation tests were performed using electromechanical and servohydraulic testing machines at $650^{\circ} \mathrm{C}$ under load control at a stress ratio of $\mathrm{R}=0.1$ in vacuum and air.

The testing program involved a variation in the frequency, the duration of dwell times in tension, and the environment (air/vacuum). Fig. 4 shows the setup of a dwell-time fatigue experiment, where the temperature was established using an induction heating system and the crack length measurements was carried out by means of an alternate current potential drop technique (ACPD).
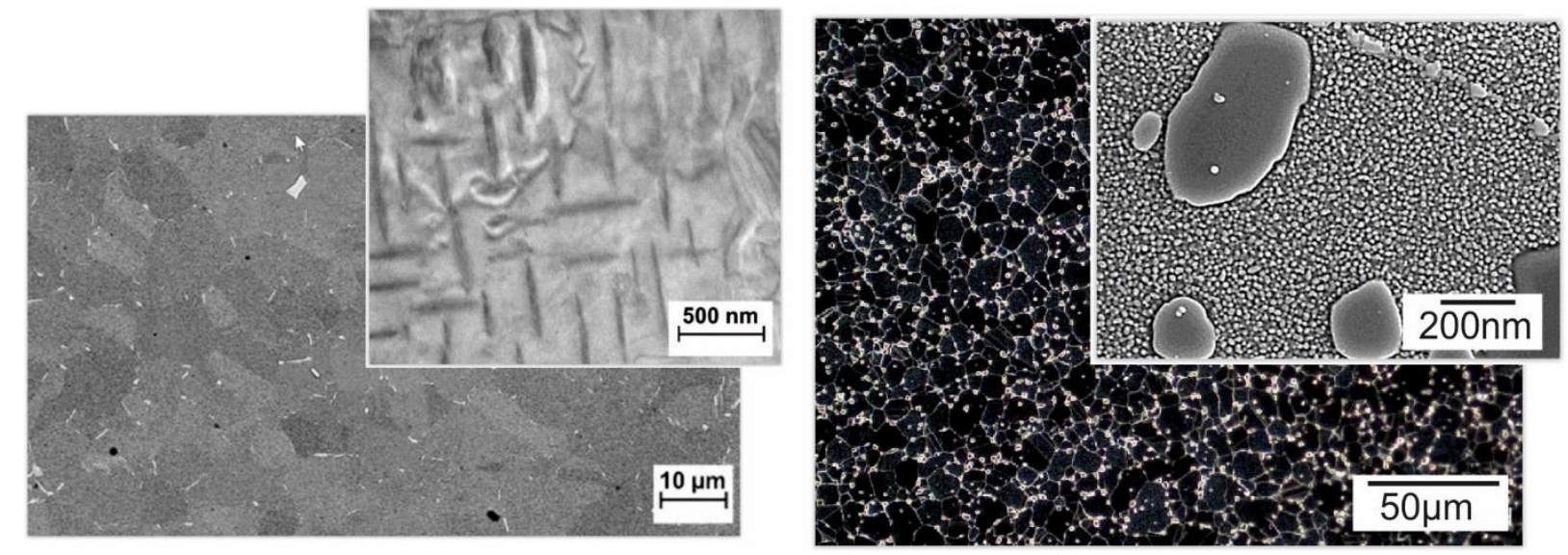

a

b

FIGURE 3. Microstructure of polycrystalline Ni-based superalloys: (a) Alloy 718 with grain boundaries pinned by $\delta$ precipitates and strengthening $\gamma^{\prime \prime}$ platelets, and (b) AD730 with large primary $\gamma^{\prime}$ precipitates pinning the grain boundaries and small spherical secondary $\gamma^{\prime}$ precipitates after ageing treatment.

corner notch $(\mathrm{CN})$ specimen

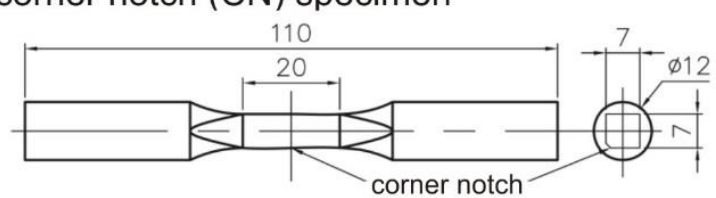

single edge notch bending (SENB) specimen

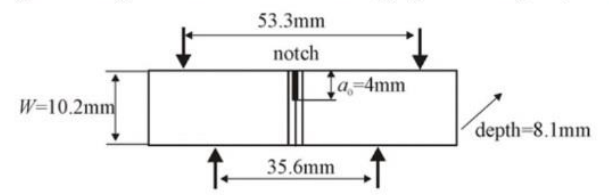

a

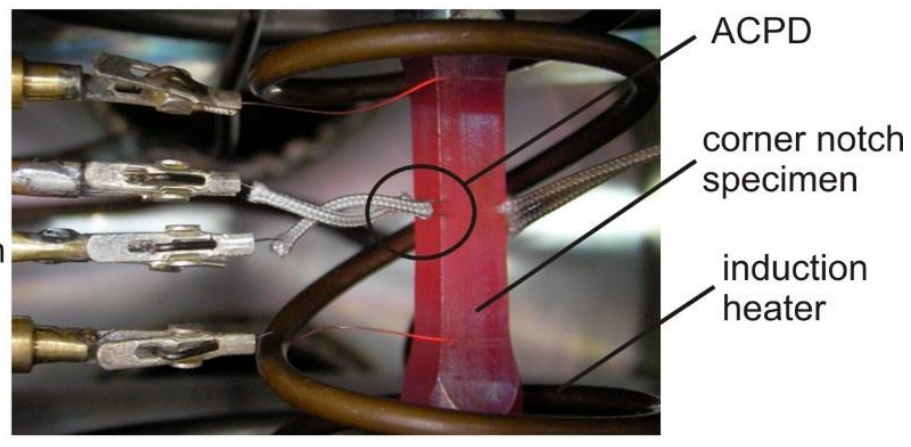

$\mathrm{b}$

FIGURE 4. Experimental set up: (a) specimen geometry of CN and SENB specimens (arrows refer to 4pt loading points), and (b) CN specimen (Alloy 718) heated by an induction coil with ACPD wires for crack monitoring.

\section{RESULTS AND DISCUSSION}

The detrimental effect of dwell times during low-cycle fatigue (LCF) of Alloy718 is represented by Fig. 5, where the crack propagation rates $d a / d N$ vs. the stress intensity range $\Delta K$ is plotted. Although the results show some scatter, the 296s dwell times in air cause an increase in the crack propagation rates by about two orders of magnitude as 
compared to $1 \mathrm{~Hz}$ and $4 \mathrm{~Hz}$ testing in air, and even more when testing under vacuum atmosphere. Having a closer look to the crack propagation vs. the time reveals that the main portion of crack advance occurs as a consequence of load reversal. This is shown in Fig. 5b. Here, the dwell-time fatigue experiment was transferred to sustained loading without load reversals to compare the respective crack-propagation response. Generally, DE sets in after a certain incubation time. In several papers, the incubation time was correlated with the formation of a damage zone ahead of the crack tip, which breaks when either a load reversal is applied or the maximum stress intensity in tension exceeds (cf. [7]).

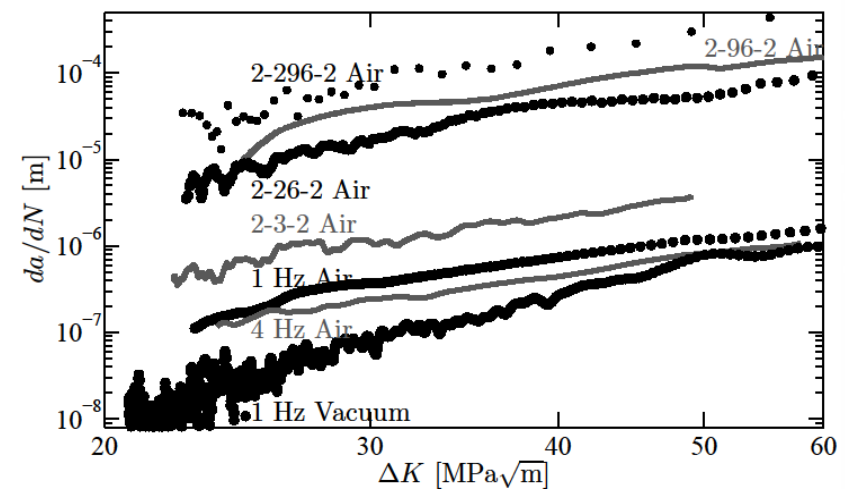

a

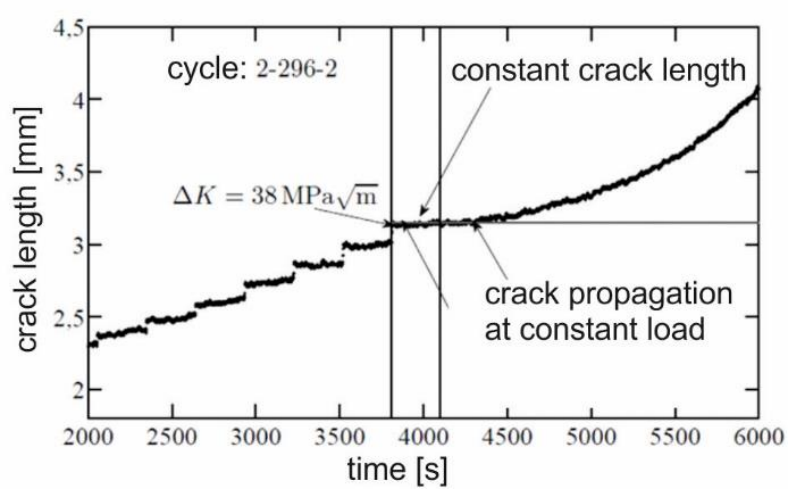

b

FIGURE 5. Fatigue crack propagation rate of Alloy 718 at $650^{\circ} \mathrm{C}$ : (a) for different test conditions as a function of the stress intensity range, and (b) crack advance vs. time: after 3800 s the test was transferred to sustained loading without load reversals.

It was found, e.g. in $[9,10]$, that intergranular cracking by DE is not a homogeneous process, but follows preferentially random high-angle grain boundaries. Therefore, the propagating intergranular crack front leaves behind uncracked ligaments that eventually fail by ductile rupture, when the local stress state exceeds the tensile strength of the material. Fig. 6 a shows an example of such an unbroken ligament. In loading scenarios with mixed blocks of dwell-fatigue cycles and pure fatigue, the ligaments formed during sustained load have been shown to crack gradually during cycling [11], which can have a large influence on the accuracy of crack length measurement. To prove the hypothesis that special CSL grain boundaries exhibit a higher resistance to DE cracking, a set of Alloy 718 samples were grain-boundary-engineering-type (GBE) thermomechanically processed (TMP: 4-times repeated sequence of cold rolling and annealing at $1050^{\circ} \mathrm{C}$, followed by ageing). By applying TMP the fraction of CSL boundaries was increased by a factor of 2 as compared to the as-received (AR) condition. Fixed-displacement load relaxation tests on SENB specimens (Fig. 6b: crack advance can be correlated with the load drop) revealed that TMP leads to (i) an increase in the incubation time until DE cracking sets in and to a decrease in the crack propagation velocity.

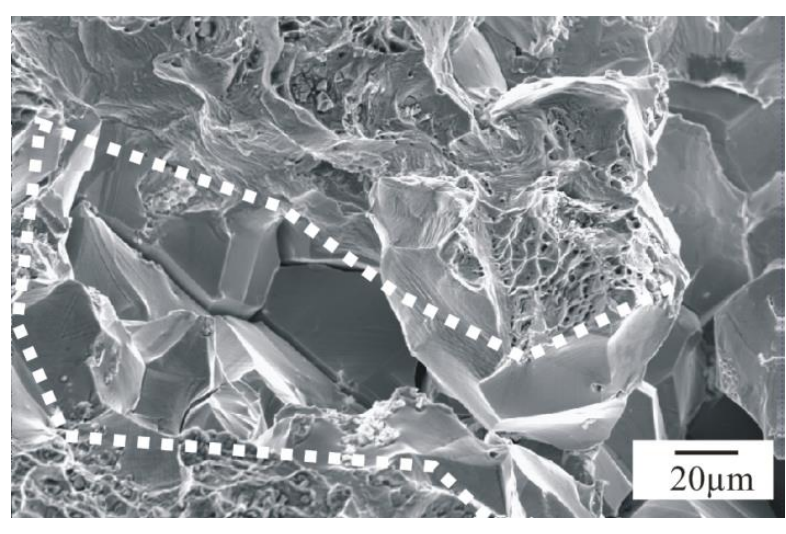

a

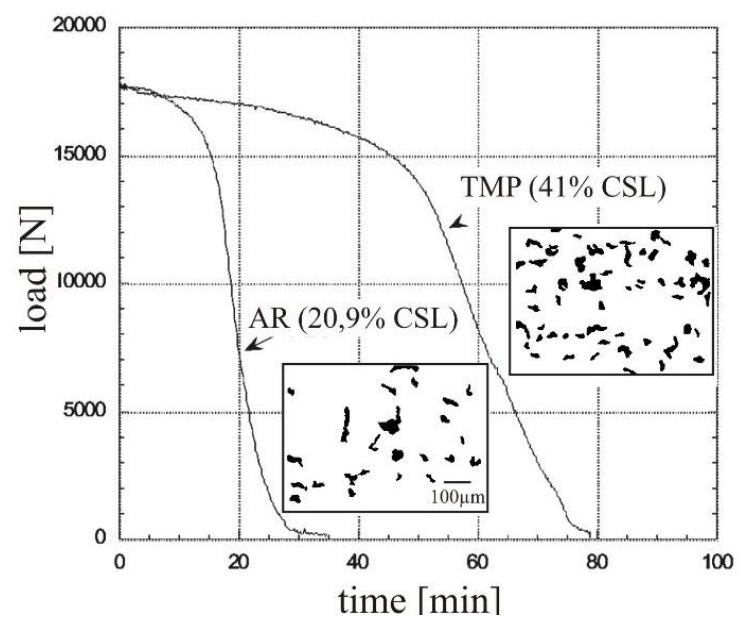

FIGURE 6. Influence of the microstructure on DE of Alloy 718: (a) ductile rupture of unbroken ligaments left behind the propagating intergranular DE crack front, and (b) load relaxation due to intergranular DE crack propagation during fixed displacement testing (cf. [1]). 
Until today, the underlying mechanism of intergranular cracking during dwell-time fatigue is controversly discussed. While the DE scheme considers nano-scale embrittler diffusion followed by continuous grain boundary decohesion, as it was shown on bicrystalline specimens $[9,10]$, the SAGBO scheme attributes crack propagation to the brittle failure of a thermally grown oxide scale along the respective grain boundaries. More recent results using transmission electron microscopy (TEM) have shown the presence of micron-sized oxide intrusions in grain boundaries ahead of growing cracks in superalloys [12,13], suggesting that the truth must lie somewhere in between. Atom probe tomography (APT) on needle-type specimens extracted from such oxides created during crack growth in isotope spiked oxygen followed by rapid cooling in Ar showed a complex layering, and the distribution of ${ }^{18} \mathrm{O}$ confirmed its formation during the dwell time [12]. It should also be noted that high resolution techniques like APT and nano-SIMS (secondary ion mass spectroscopy) have confirmed that no detectable transport of oxygen into the material takes place in the plastic zone at the crack tip [10].

Nevertheless, the DE scheme seems to be the most probable one to explain intergranular crack propagation rates as high as $d a / d t=50 \mu \mathrm{m} / \mathrm{s}$; much too little time for the formation of a massive TGO. According to TGA measurements, the parabolic rate constant of $\mathrm{Cr}_{2} \mathrm{O}_{3}$ was determined as $k_{\mathrm{p}}{ }^{\prime \prime} \cong 8.7 \cdot 10^{-14} \mathrm{~g}^{2} \mathrm{~cm}^{-4} \mathrm{~s}^{-1}$ at $650^{\circ} \mathrm{C}$. Hence, only the formation of just monolayers should be possible during the exposure of fresh metal surface at a DE crack propagation rate of $50 \mu \mathrm{m} / \mathrm{s}$. While crack propagation by DE is continuous during $4 \mathrm{pt}$ bend loading of a single grain boundary in a bicrystalline specimen, it tends to be discontinuous in a polycrystalline material. However, the respective damage zone is rather nano-scale and could be identified as small striation-like features in the fracture surface (see Fig. 7b). It is worth mentioning that superalloys being sub-solvus solutionized and hence, exhibiting a fine-grained microstructure (cf. section Materials and Experimental) are not prone to intergranular cracking by DE. This can be attributed to a pronounced crack tip shielding effect due to plasticity (fine grained materials is softer) or crack deflection.

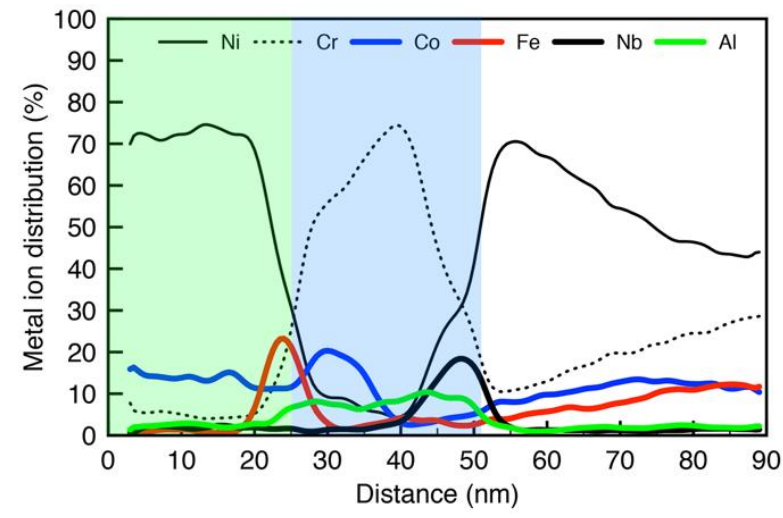

a

(b) striation-like features within the intergranular grain-boundary facets, resembling non-discontinuous DE.

\section{MODELING SCOPE FOR DWELL-TIME FATIGUE}

Today, fatigue life assessment for structures exposed to dwell-time LCF is based on semi-empirical Coffin/Manson approaches following equ. 2, or concepts based on the exponential Paris law (cf. equ. 1) for fatigue crack propagation. From experimental work, a pronounced mutual relationship between cycle and time-dependent damage behavior became obvious. This led to the concept of the damage zone; cf. [7,8]. Since an increase in the dwell time interval and a decrease in the stress intensity range led to an increase in the fraction of intergranular DE attack (Fig. 8a), the LCF crack propagation rate as a function of mechanical load and dwell time can be written as follows:

$$
\frac{d a}{d N}\left(\Delta K, K_{\max }, t\right)=b \sqrt{D_{\text {eff }}\left(K_{\max }\right) t} M+(1-b) C_{\text {trans }} \Delta K^{n_{\text {trans }}},
$$

where $D_{\text {eff }}$ denotes an effective grain boundary diffusion coefficient of oxygen, which is expressed as a function of $K_{\max }$ (in the 296s reference dwell test, crack propagation is exclusively intergranular). For the transgranular crack growth contribution the Paris law (cf. equ. 1) is applied with the constants $C_{\text {trans }}$ and $m_{\text {trans }}$ as determined from the crack 
propagation test at $1 \mathrm{~Hz}$ in vacuum (no DE). The parameter $M$ represent the dwell time fraction of the experiment, while $b$ denotes the intergranular part of the fracture surface (experimentally determined, cf. Fig. 8a). The respective experimental and simulated crack propagation rates (Fig. 8b) are in reasonable agreement supporting the damage zone concept in general.

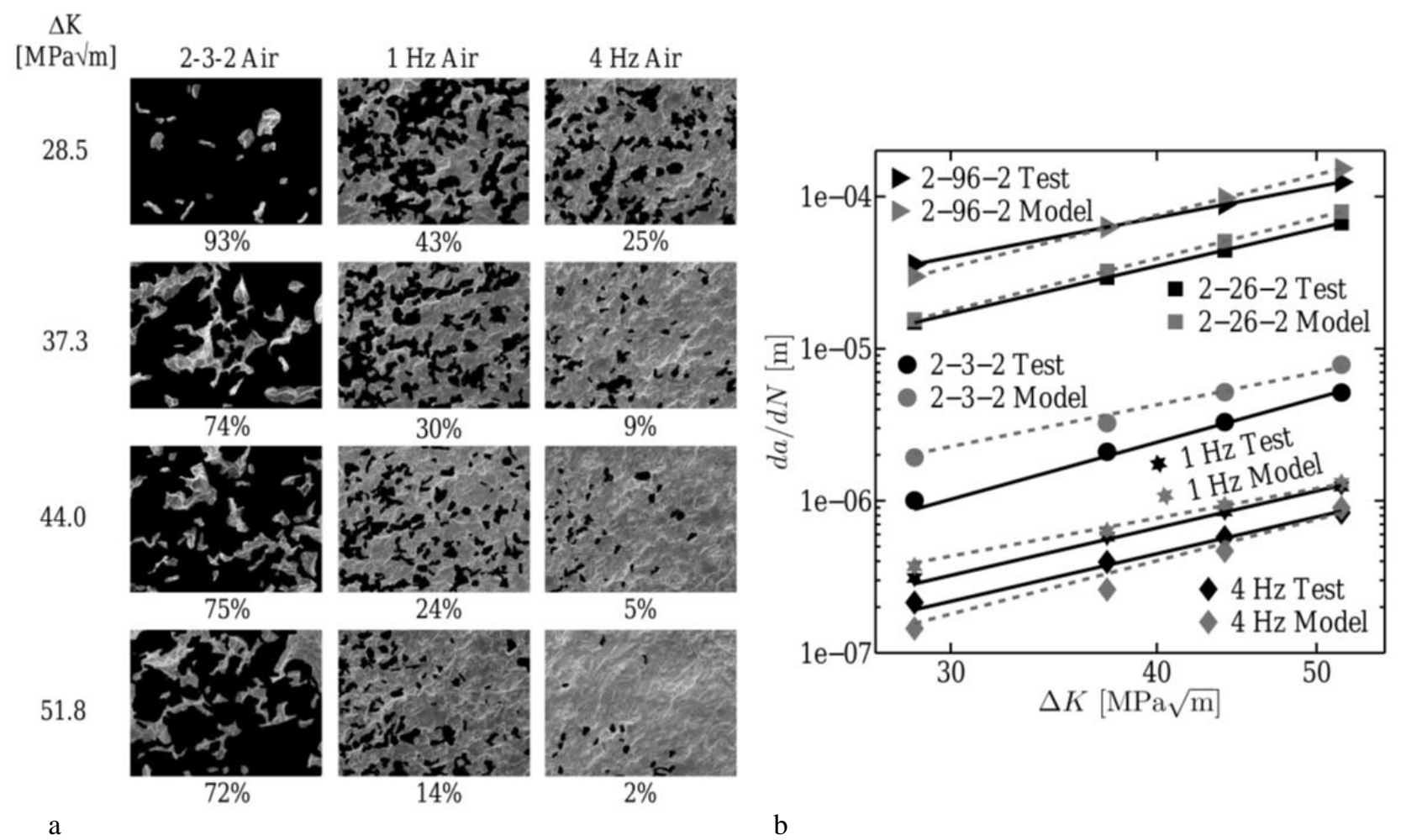

FIGURE 8. (a) Quantitative evaluation of the intergranular part of the fracture surface vs. the stress intensity range

$\Delta K$ for three different fatigue tests at $650^{\circ} \mathrm{C}$ and a stress ratio of $R=0.1: \mathrm{f}=1 \mathrm{~Hz}, \mathrm{f}=4 \mathrm{~Hz}$ and $3 \mathrm{~s} \mathrm{dwell} \mathrm{time} \mathrm{at}$ maximum load, and (b)comparison of experimentally observed crack propagation rates with calculated growth rates for different test conditions.

However, a sound prediction of the DE crack propagation rate for various alloy microstructures requires the implementation of stress-assisted grain boundary diffusion $\left(D_{\mathrm{gb}}\right)$ and interfacial separation within the cohesive zone ahead of the crack tip:

$$
\frac{d c}{d t}=D_{g b} \frac{\partial^{2} c}{\partial x^{2}}+\frac{D_{g b} \Omega}{k T} \frac{\partial}{\partial x}\left(c \frac{\partial \sigma}{\partial x}\right),
$$

with $k$ being the Boltzmann constant, and $\Omega$ the atomic volume. Knowing the actual stress state, e.g., as a result from grain-structure-based finite element simulation (accounting for elastic anisotropy) of the resolved tensile stress state $\sigma$ acting on the alloy grain boundaries (cf. Fig. 1), equ. 4 (simplified for on-dimensional diffusion along the grain-boundary plane) can be solved and used to calculate the embrittler concentration $c$. Since intergranular decohesion is governed by a critical combination of tensile tractions and embrittler concentrations, time-dependent crack propagation according to DE should be predictable. This is subject of ongoing research work. In an earlier study [14], a simplified but analytical treatment of the DE mechanisms yield the following expression for the embrittler concentration at a distance $x$ ahead of the crack tip:

$$
c=c_{0} \exp \left[-\frac{v}{D_{\mathrm{gb}}} x+\frac{\Omega}{k T}\left(\sigma-\sigma_{0}\right)\right] .
$$


When applying equ. 5 to DE of alloy 718, one obtains a steep decrease in the embrittler concentration within a few nanometers, supporting the hypothesis that DE is an oxygen-diffusion-controlled process being active in the close vicinity of the crack tip without formation of a compact oxide layer.

\section{CONCLUSIONS}

Polycrystalline Ni-based superalloys designed for dynamically high-loaded structures, e.g., as gas turbine discs, tend to intergranular cracking when exposed to an oxidizing environment. According to the dynamic embrittlement scheme, oxygen penetrates grain boundaries that are (i) exposed to high tensile traction, and that (ii) exhibit a rather open structure (high angle random grain boundaries). Diffusion is followed by interfacial decohesion ranging a few nanometers ahead of the crack tip. At intermediate remote stress intensities, the separated grain boundaries are shielded by non-broken grain boundaries (e.g., low-energy CLS boundaries) until the stress intensity exceeds a critical value for ductile rupture of the remaining boundaries. The increase of the local stress intensity can be attributed either to creep or to load reversal during dwell-time fatigue. Investigations of the local chemistry in the close vicinity of the crack tip by atom probe tomography revealed the formation of transition oxides. However, no compact oxide scale was identified ahead of the crack tip. Therefore, the hypothesis of a nanometer-scale embrittling zone is more likely than a large "damage zone". The latter one can be understood as the area containing a mixture of separated and intact grain boundaries. A more detailed analysis by a microstructure-based diffusion-decohesion assessment is subject of ongoing work by the authors.

\section{ACKNOWLEDGMENTS}

The financial support by Deutsche Forschungsgemeinschaft (DFG), the Volkswagen Foundation, the Alexander von Humboldt foundation, the Swedish Innovation Agency (VINNOVA), the Swedish High Temperature Corrosion Centre (HTC) and The Swedish Foundation for Strategic Research (SSF) is gratefully acknowledged by the authors.

One of the authors (UK) wants to express his deep gratitude to Prof. Charles J. McMahon Jr. for the collaboration and plenty of fruitful discussions not only on dynamic embrittlement.

\section{REFERENCES}

1. U. Krupp, Intern. Mater. Rev., 50, 83 (2005).

2. A. Pineau and S.D. Antolovich S.D, Engng Failure Anal., 16, 2668 (2009).

3. J.A. Pfaendtner and C.J. McMahon Jr, Acta Mater., 49, 3369 (2001).

4. M.F. Browning, and M.F. Henry, Proc. Superalloys 718, 625 and Various Derivates, E.A. Loria (eds.), TMS (1997) 665.

5. K. Löhnert, doctorate thesis, Friedrich Alexander University Erlangen-Nürnberg 2011.

6. W.J. Ostergreen, Journal of Testing and Evaluation, 4, 327 (1976).

7. E. Lundström, K. Simonsson, D. Gustafsson and T. Månsson, Engng Fracture Mech., 118, 17 (2014).

8. K. Wackermann, doctorate thesis, University of Siegen 2014.

9. U. Krupp, J. Mater. Sci., 43, 3908 (2008).

10. W. Kane, PhD thesis, University of Pennsylvania, Philadelphia 2005.

11. M. Hörnqvist, L. Viskari, K.L. Moore, Y. Cao and K. Stiller, Mater. Sci. Eng. A, 609, 131 (2014).

12. L. Viskari, M. Hörnqvist, K.L. Moore, Y. Cao and K. Stiller, Acta Mater., 61, 3630 (2013).

13. H.S. Kitaguchi, H.Y. Li, H.E. Evans, R.G. Ding, I.P. Jones, G. Baxter, P. Bowen, Acta Mater., 61, 1968 (2013).

14. Y. Xu and J.L. Bassani, J.L., Mater. Sci. Enging, A260, 48 (1999). 